\title{
ESD testing and combdrive snap-in in a MEMS tunable grating under shock and vibration
}

\author{
Subramanian Sundaram ${ }^{\mathrm{a}, \mathrm{b}}$, Maurizio Tormen ${ }^{\mathrm{c}}$, Branislav Timotijevic ${ }^{\mathrm{c}}$, Robert Lockhart ${ }^{\mathrm{c}}$, Ross P. \\ Stanley ${ }^{\mathrm{c}}$, Herbert R. Shea ${ }^{\mathrm{a}}$ \\ ${ }^{a}$ Ecole Polytechnique Fédérale de Lausanne, rue Jaquet Droz 1, 2002 Neuchâtel, Switzerland \\ ${ }^{\mathrm{b}}$ Birla Institute of Technology and Science, Pilani, Rajasthan - 333031, India \\ ${ }^{c}$ Centre Suisse d'Electronique et de Microtechnique, rue Jaquet Droz 1, 2002 Neuchâtel, Switzerland
}

\begin{abstract}
This work describes a method for tracking the dynamics of electrostatic discharge (ESD) sensitive MEMS structures during ESD events, as well as a model for determining the reduced combdrive snap-in voltage under vibration and shock. We describe our ESD test setup, based on the human body model, and optimized for high impedance devices. A brief description of the MEMS tunable grating, the test structure used here, and its operation is followed by results of the measured complex device dynamics during ESD events. The device fails at a voltage up to four times higher than that required to bring the parts into contact. We then present a model for the snap-in of combfingers under shock and vibration. We combine the results of the analytical model for combdrive snap-in developed here with a shock response model to compute the critical shock acceleration conditions that can result in combdrive snap-in as a function of the operating voltage. We discuss the validity regimes for the combdrive snap-in model and show how restricting the operation voltage below the snap-in voltage is not a sufficient criterion to ensure reliable operation especially in environments with large disturbances.
\end{abstract}

Keywords: ESD testing, combdrive snap-in, reliability model, tunable MEMS grating

\section{INTRODUCTION}

Reliability estimation of MEMS devices has been mainly done thorough extensive Electrostatic discharge (ESD) tests ${ }^{1,2}$, shock and vibration tests ${ }^{3,4}$, fatigue and creep tests ${ }^{5,6}$, and aging tests through rapid thermal cycling. These tests have so far mainly been used to qualify devices. Though the current understanding of the physics of reliability is evolving quite rapidly with the availability of advanced techniques and tools, predicting the conditions for failure before hand is still a very challenging problem. This problem is further elevated by the presence of various complex failure mechanisms for a generic MEMS device 7 . Many previous works on reliability testing are based on inspecting failed devices to understand the failure mechanism and thresholds, as in the case of ESD testing, or consider conditions where the devices operate in a steady or stable environment. Our goal is to focus on the importance of measuring the dynamics of failure events as a part of reliability testing.

A specific optical MEMS device, a tunable grating ${ }^{8,9}$, has been adopted as a test vehicle to analyze the transient behavior of two failure mechanisms; first, the transient behavior during ESD events, then the failure of combdrive structures under shock and vibration during normal actuation conditions. The knowledge of transient behavior can be used to improve the device design and its resistance to failures.

The classification of commercial devices based on the sensitivity to ESD has been in practice for a very long time. Estimation of the reliability with respect to ESD events is important since any device is always subjected to minor inputs at all stages of the lifecycle, from fabrication to handling. While ESD failures are mostly dominated by the breakdown of materials, especially various oxides, in the case of semiconductor devices, in MEMS devices a number of other possible failure mechanisms such as stiction and micro-welding can be found. So far ESD testing has mainly been used to obtain the statistics of failed devices, after the end of the ESD event, and to estimate the effects of failure ${ }^{1,2}$; to date, only few transient behavior studies have been reported, e.g. the dynamics of a RF MEMS switch during ESD failure has been tracked using a Laser Scanning Vibrometer (LSV) ${ }^{10}$.

Reliability, Packaging, Testing, and Characterization of MEMS/MOEMS and Nanodevices X, edited by Sonia Garcia-Blanco, Rajeshuni Ramesham, Proc. of SPIE Vol. 7928, 79280A (C) 2011 SPIE · CCC code: 0277-786X/11/\$18 - doi: 10.1117/12.873253

Proc. of SPIE Vol. 7928 79280A-1 
In this work, we use a Human Body Model (HBM) based tester integrated with a Laser Doppler Vibrometer (LDV) to track the dynamics for a range of ESD voltages showing how the transient displacement evolves with increasing ESD voltages in extremely sensitive modes of our device.

The second part of our work describes an analytical model for the conditions leading to combdrive snap-in at operating voltages much below typical snap-in voltages recorded in a mechanically steady state. This is mainly motivated by the need for developing robust devices that that can safely withstand mechanical inputs such as shocks. Since combdrives account for a major part amongst all micro-actuators, it becomes essential to understand the effects of mechanical inputs on the failure conditions for combdrives. We show how the snap-in voltage can be reduced considerably in the presence of mechanical disturbances. For example, our models and experimental tests show that the lateral snap-in of combdrives occurs at lower voltages, passing from $97.5 \mathrm{~V}$, under mechanically undisturbed conditions, to $67 \mathrm{~V}$, in the presence of $1000 \mathrm{~g}$ axial shocks. We now introduce the tunable MEMS grating, which has been used for our testing procedures.

\section{MEMS TUNABLE GRATING AND ITS FAILURE MODES}

A MEMS tunable grating is a device that acts as an optical tunable filter, when it is actuated. The tuning is obtained by stretching the grating array in the grating plane ${ }^{8,9}$. The device considered here was manufactured by the CSEM (Switzerland). It is fabricated from an SOI wafer ( $10 \mu \mathrm{m}$ single crystal Si device layer on top of a normally $2 \mu \mathrm{m}$ thick $\mathrm{SiO}_{2}$ layer), using a single mask process to define the structure in the device layer of the SOI wafer ${ }^{8,9}$. The grating consists of an array of suspended rectangular beams, $10 \mu \mathrm{m}$ high, interconnected by folded beam structures that act as springs. The two sides of the grating are attached to electrostatic combdrive actuators that are used to tune the gap between the adjacent beams. On the application of a voltage across the two sets of combdrives, the grating expands such that the gap between the adjacent beams increases uniformly and is regulated by the stiffness of the interconnecting springs. To ensure uniformity and reduce the possibilities of an offset due to the mismatch in the combdrives on the two sides, the central beam of the grating is fixed rigidly. Initially, the $9 \mu \mathrm{m}$ wide beams are uniformly positioned with a period of $13 \mu \mathrm{m}$. Figure 1(a) shows a schematic model of one half of the grating, where the hatched regions denote the fixed anchors. The plane ' $\mathrm{S}$ ' represents the surface of symmetry. In the figure, only the mobile part of the combdrive is shown. As shown in the figure 1(a), under normal conditions of operation, the grating stretches in the plane of the grating ( $y$ direction). Detailed analysis of reliability tests is reported in our recent work ${ }^{11}$.

In this work, we only focus on two specific failure modes that are relevant here. The first mechanism that we consider here is the out-of-plane failure of the grating. The gap between the grating and handle layer lies between $1.6 \mu \mathrm{m}$ and 2 $\mu \mathrm{m}$, significantly smaller than the in-plane dimensions of the structure $(1 \mathrm{~mm} \times 1 \mathrm{~mm})$. This makes the out-of-plane mode of the structure particularly failure prone: snap-in, in fact, occurs at a voltage of $0.96 \mathrm{~V}$ in this mode. Figure 1(b) shows a schematic of the out-of-plane mode in half of the resonant mode cycle where the grating beams deflect downwards (negative $z$ direction) and this may possibly lead to contact with the underlying substrate layer and thereby to stiction.

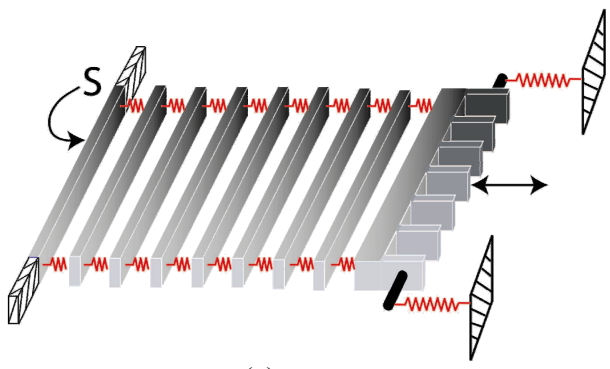

(a)

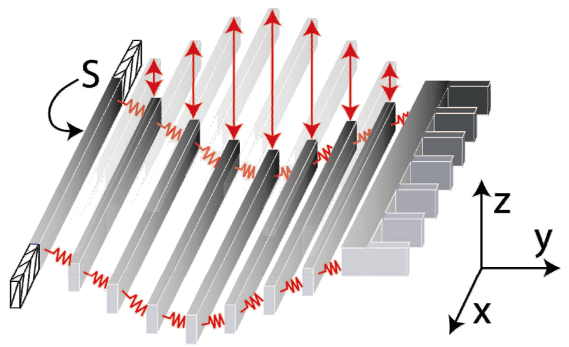

(b)

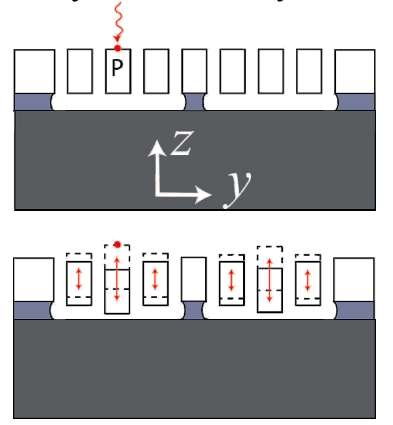

(c)

Figure 1. Schematic representation of one half of the grating: ' $S$ ' indicates the plane of symmetry and the hatched regions show the fixed parts. (a) Normal mode of operation where the grating expands as a function of the applied voltage. This is also the important dynamic in-plane mode that decides the dynamic range of operation. (b) Schematic of the out-of-plane mode shape. (c) Schematic cross section of the entire grating in a steady state and a view of the out-of-plane mode. The clamped central beam is also shown. The Laser Doppler Vibrometer (LDV) measurements are made at the point ' $\mathrm{P}$ ', which is the position with the largest displacement in the structure. 
Figure 1(c) shows the cross section of the grating in the steady state and a schematic of the out-of-plane mode. This mode is the most critical one; its undamped natural frequency, obtained in high vacuum $(<1 \mathrm{mbar})$, is $2.36 \mathrm{kHz}$. Due to the small gap between the device layer and the handle layer, the device is overdamped in its out-of-plane mode providing an improvement in terms of reliability. The quality factor of this mode in air is found to be 0.382 .

The second failure mechanism, analyzed here is the lateral snap-in of the electrostatic combdrive actuators. The resonant mode that corresponds to cycles of uniform expansion and compression of the grating is the important mode here. The natural frequency and the equivalent quality factor seen at the combdrive end are found out to be $3.63 \mathrm{kHz}$ and 57.47 respectively using the strobed illumination mode of a white light interferometer (WLI). In response to the application of a DC voltage across the combdrives, the displacement seen at the combdrives can be used to calculate an equivalent spring stiffness (or compliance) of the structure. The spring compliance (with respect to the applied voltage) is found to be $2 \mathrm{~nm} / \mathrm{V}^{2}$. The typical lateral snap-in voltage is around $97.5 \mathrm{~V}$ (under mechanically undisturbed conditions) for the combdrive actuators.

\section{TRANSIENT BEHAVIOUR OF MEMS TUNABLE GRATINGS DURING ESD EVENTS}

ESD failures are a common route for device failures and hence testing methods and standards have been devised to facilitate the use of a uniform classification scheme based on ESD sensitivity ${ }^{12}$. All devices that fail for less than $250 \mathrm{~V}$ ESD events are grouped in class 0 , which is the most sensitive class. As will be shown later, our devices fail during ESD zaps of even less than $10 \mathrm{~V}$. The entire classification scheme can be found from in ANSI's ESD standards ${ }^{12}$. In the case of integrated circuits (ICs), ESD protection circuits can be conveniently placed in the path between the external contact pins and the transistors that implement the specific functionality of the IC. While a similar solution may suffice for MEMS integrated with the control electronics monolithically, very little knowledge is available on the ESD failure mechanisms and levels of stand alone MEMS structures.

In order to approximate the discharging process from a human body, the Human Body Model (HBM) is commonly used to test the device. Other models like the Machine Model (MM) are also used to model events where machine parts act as the discharge source. Due to the usually large resistance of electrostatic MEMS, the results of tests with the MM and the HBM are very similar ${ }^{1}$.

We constructed a HBM based ESD tester that provides a synchronization trigger to a Laser Doppler Vibrometer (LDV) at the start of the ESD event. In short, an LDV tracks the Doppler shift in the frequency of the Laser after it is reflected from a moving point, to measure the instantaneous velocity of the point. This velocity information can also be recorder at an array of points, and processed further to generate the displacement data. In our case, the velocity was measured continuously at the point ' $\mathrm{P}$ ', on the top surface of the finger with the largest displacement, as shown in figure 1(c).

Figure 2(a) shows the circuit diagram of the HBM tester. The conventional HBM consists of a 100pF capacitor discharging to the device under test through a $1500 \Omega$ resistor. The two relays $S_{1}$ and $S_{2}$, which are rated for high voltages, are used to control the charging and discharging cycles. The typical operation involves two steps. First, the relay $S_{1}$ is kept closed till the voltage across $C_{\mathrm{HBM}}$ reaches the desired ESD test voltage. The next step involves simultaneously opening $S_{1}$ and closing $S_{2}$. The latter step is crucial with three main requirements: (1) The whole process must be quick enough to prevent the charge across $\mathrm{C}_{\mathrm{HBM}}$ from leaking to ground through alternate pathways (2) It has to be ensured that there is never a direct path between the supply voltage and the test device, and (3) The occurrence of jitters has to be avoided. This control of $\mathrm{S}_{1}$ and $\mathrm{S}_{2}$ was done by using a TTL circuit with a tunable delay between signals arriving at $\mathrm{S}_{1}$ and $\mathrm{S}_{2}$.

A block diagram of the overall setup of the tester, LDV and the test structure is shown in figure 2(b). The custom built ESD tester mainly consists of two parts: the control circuitry and the HBM circuit. The control circuitry, as discussed earlier, is used to provide tunable delays between the relays, which essentially has to account for the time difference between the make ( $\sim 3-4 \mathrm{~ms}$ for our relay) and break ( $\sim 1 \mathrm{~ms}$ for our relay) response times in the relay. The control circuit, the heart of our ESD tester, was also designed such that a trigger output can be used to synchronize the LDV measurement and the ESD event. 


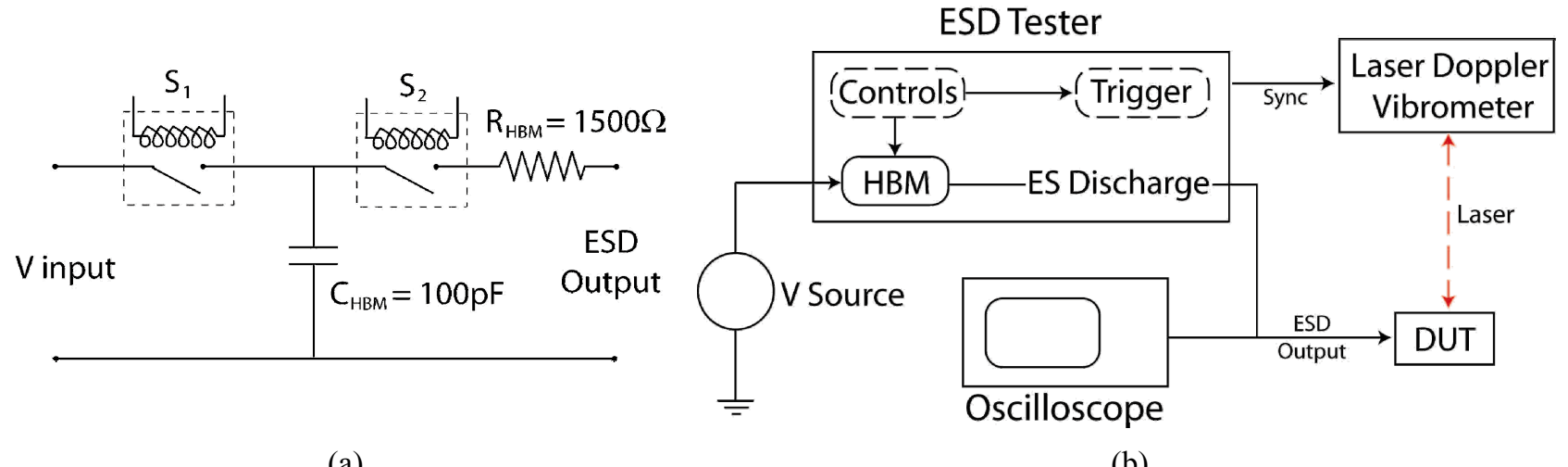

(a)

(b)

Figure 2. (a) Schematic of the Human Body Model (HBM) used for ESD testing (b) Complete setup of the system used to capture the displacement transients during ESD events.

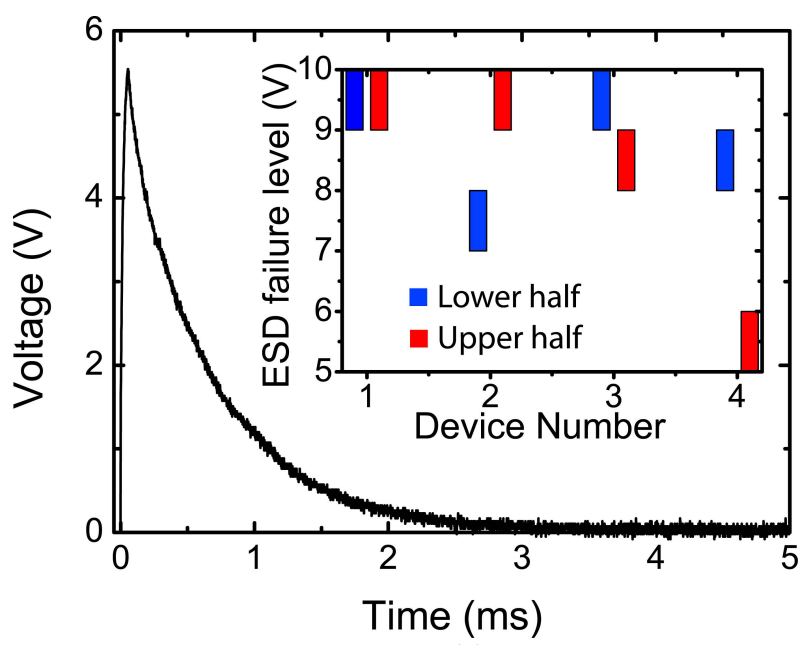

(a)

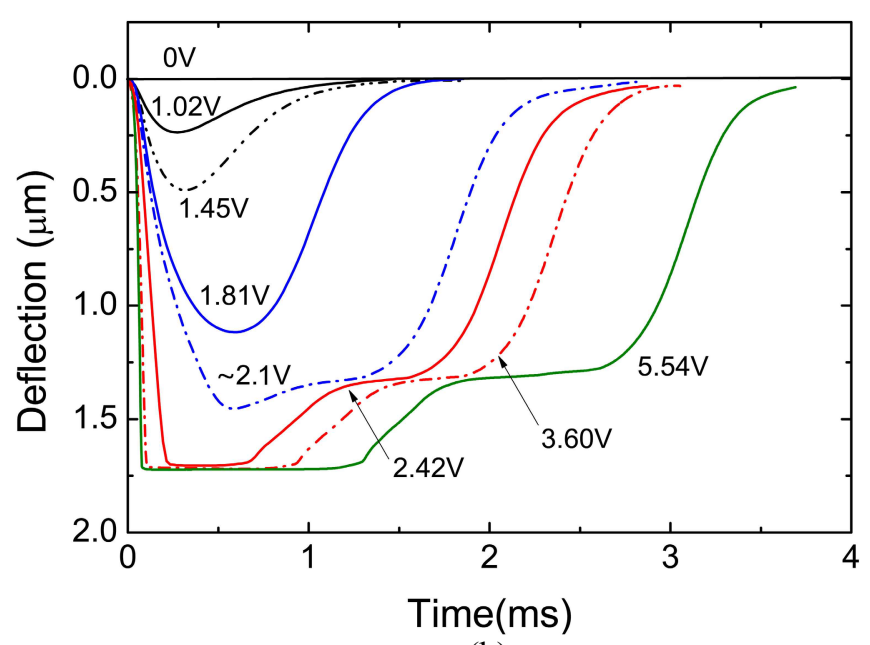

(b)

Figure 3. (a) A typical discharge waveform observed during ESD tests. This particular sample was recorded during a $6 \mathrm{~V}$ zap on device no. 2(Inset). Inset shows the ESD levels that lead to failures for 4 different test devices. It can be seen that all devices have failed by $10 \mathrm{~V}$ ESD zaps. The blue and red regions show the failure levels for two independent halves of the grating. (b) Recorded displacement waveforms observed on a specific device for different test levels.

We were motivated to study the ESD failure levels and the displacement transients for our device, which very clearly seemed to be sensitive to ESD. The most sensitive mode in the tunable grating with respect to ESD is the out-of-plane mode, shown in figure 1(b). This is mainly due to the large in-plane dimensions and small a separation $(\sim 1.7 \mu \mathrm{m})$ between the device layer and handle layer. The mass of the structure is considerably high due to the in-plane dimensions (order of $1 \mathrm{~mm}$ ) and moreover, the spring compliance is also high for the out-of-plane mode since the inter-finger springs are connected in series. Therefore, small accelerations or applied voltages (approx $1 \mathrm{~V}$ between the grating and the handle layer ${ }^{11}$ ) can cause the grating to deflect, as shown in figure 1(b), and touch the underlying substrate. Contact between the grating and the handle layer can lead to failure by stiction if the contact surfaces are smooth. However, our devices clearly show that the contact roughness is sufficiently large to prevent the occurrence of stiction ${ }^{11}$. During ESD failures through this mode, contact rather leads to the formation of micro welding between the two layers. It is worth noting that the pull-in and pull-out DC voltages of this mode are $0.96 \mathrm{~V}$ and $0.27 \mathrm{~V}$ respectively ${ }^{11}$. Other possible ESD failures in our device can result from discharges across the combdrives but this is expected to occur at a large voltage since the snap-in voltage is itself of the order of $\sim 100 \mathrm{~V}$. 
We performed ESD tests on the out-of-plane mode by applying the discharge waveform across the device layer and the handle layer. A typical ESD waveform seen across the device (between the grating and the substrate) for a $6 \mathrm{~V}$ zap is shown in figure 3(a). This particular waveform is recorded from a device that was still functional after the ESD test. The discharge time scale is of the order of milliseconds due to the large resistance of the device under test (DUT). A device that fails by short-circuit would have a much shorter pulse. During each of the ESD tests, the trigger output from the ESD tester, was used to initiate the velocity measurement in the LDV. The velocity was measured as a function of time at the finger with the largest displacement in each half of the grating. The small linear offset due to noise in the velocity transient was eliminated and this processed waveform was integrated to obtain the actual displacement of the point as a function of time. Figure 3(b) shows the measured displacement transients during various ESD tests on a single device. The peak discharge voltage is indicated next to each curve. The ESD failure levels were recorded for the two symmetric halves of the grating, increasing the discharge voltage in steps of $1 \mathrm{~V}$. The inset of figure 3(a) shows the recorded failure ESD levels of 4 different devices. All devices fail at or below $10 \mathrm{~V}$ zaps. The failures due to these ESD tests could be easily seen using the optical microscope or analyzed using an optical surface profiler.

In figure 3(b), for peak ESD voltages up to $1.8 \mathrm{~V}$ appearing across the grating and the handle layer, the downward displacement of the most compliant beam (point ' $\mathrm{P}$ ' in figure 1(c)) sharply increases and return to the initial position after $\sim 2 \mathrm{~ms}$, as expected for an overdamped system, where the peak velocities are limited by the damping mechanisms predominantly by squeeze film damping in this case. No failures are seen. Surprisingly, as the peak ESD voltage is increased, a new stable plateau emerges at a displacement of $1.3 \mu \mathrm{m}$. The reason for the appearance of a stable noncontact displacement level is still not clear and needs further investigations. On increasing the voltage further beyond $2.4 \mathrm{~V}$, the central grating beam starts colliding with the handle layer and remains stationary there until the instantaneous voltage drops below a particular threshold voltage. This is seen as a horizontal line in figure 3(b) at a displacement of $\sim 1.7 \mu \mathrm{m}$ (ordinate), which corresponds to the gap between the grating and the handle layer. On increasing the test voltage, the duration of contact increases and the response after release is nearly identical (see curves for $2.42 \mathrm{~V}, 3.60 \mathrm{~V}$ and $5.54 \mathrm{~V})$. No permanent stiction is seen until the ESD voltage exceeds a failure threshold, proving that the contact roughness is sufficiently large to prevent the occurrence of stiction ${ }^{11}$. Beyond the failure threshold, a micro-welding defect occurs and prevents the grating from returning to its normal position.

By continuously recording the displacement transients during the ESD event, a detailed analysis of failure mechanisms can be performed. In fact, a permanent failure would appear only for ESD levels of around 10V, but transients for lower levels have shown how the displacement curves are different from what would normally be expected and how a simple two step contact-release process cannot account for the emergence of a new stable plateau. Moreover, the dynamic measurements show that in the specific case, ESD levels as low as $2.5 \mathrm{~V}$ do not result in a failure, even if device layer and substrate come into contact. This is the case thanks to the rough surface of the substrate; with a reduced surface roughness, ESD failures may occur simply by stiction and for voltage levels smaller by a factor of 4 .

The proposed method thus provides us ample information in the form of a clear threshold when contact is first created, the failure mechanism and other conditions that can lead to failures below currently measured failure levels. The same test method, if applied to an array of points on the device structure, can help in visualizing the dynamics through an ESD event mode. This may particularly be useful in identifying the first device part that initiates failure through contact, especially in complex devices. This information makes it possible to design appropriate stoppers and other mechanisms to retard failures. Predicting ESD failures is difficult since many processes occur simultaneously. For instance, different parts of a MEMS structure may move, charges may decay through a change in the capacitance or through leakage paths, and the force acting on different parts itself is a function of the instantaneous voltage and the actual position of the part. Under these conditions, analytical solutions may be impossible for complicated structures. Transient measurements made during the ESD event offer on one hand, help in identifying the first appearing failure modes and thus help in refining the design; on the other hand they offer a possibility to develop phenomenological models of the device under investigation.

\section{COMBDRIVE FAILURES ACCELERATED BY SHOCK AND VIBRATION}

Combdrives are key components in many commercial MEMS devices, as they allow electrostatic actuation without pullin in the actuation direction which is seen in parallel plate actuators. There is however a lateral pull in for comb-drives, where the comb snaps-in perpendicular to the actuation direction (see figure 4). 

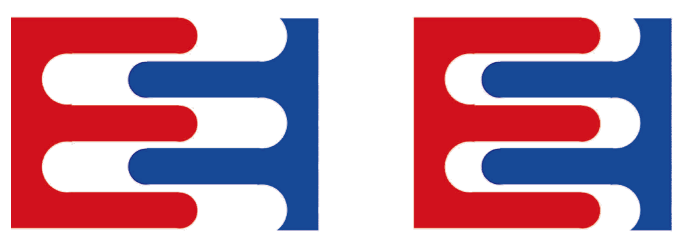

(a)
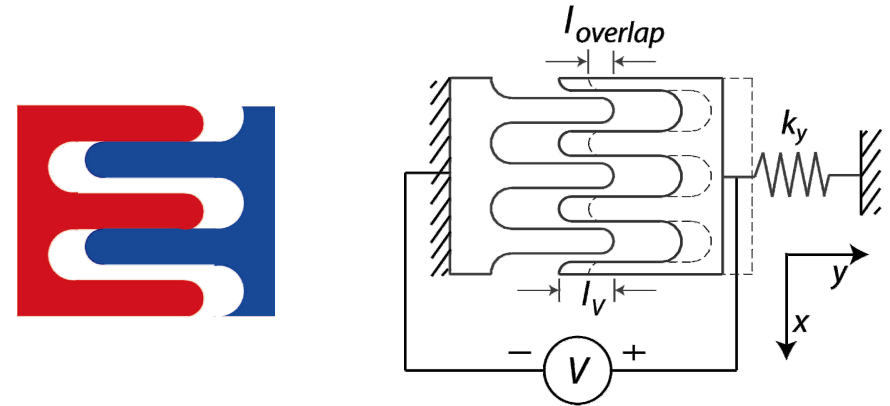

(b)

Figure 4. (a) An illustration of the mechanism of lateral snap-in occurring during operation below the normal snap-in voltages: (i) Normal position of the fingers of the combdrive at a given voltage (ii) Additional overlap created by a mechanical disturbance (iii) Snap-in at a voltage lower than the normal snap-in voltage, due to the presence of extra comb finger overlap that forces the structure into a laterally unstable domain. (b) Schematic of the combdrive showing the parameters used in the model.

The tuning of the diffraction grating has a quadratic dependence on the voltage applied at the combdrives. The tuning

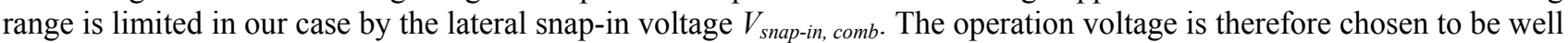

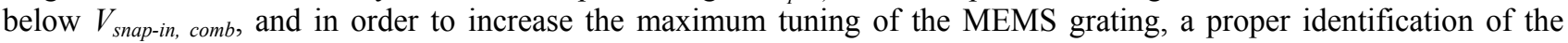
snap-in voltage becomes crucial.

Here, we investigate in particular, the impact of vibration and shock on the snap-in voltage of combdrives. To address this problem, we study how snap-in is initiated and then extend the model to include external mechanical contributions that lead to snap-in at much reduced voltages.

In an ideal combdrive, the actual lateral force is always balanced from both sides, and there is no net lateral force. Under a small lateral perturbation, the combdrive experiences a nonzero lateral force. When the net lateral force across the combdrive increases beyond the maximum reaction force supported by the anchor springs, the structure becomes unstable. This snap-in voltage is influenced by various parameters such as the combdrive geometry, uniformity of the gap between the fingers and the surface finish on the vertical walls, which is determined by the fabrication process.

The lateral force required to cause snap-in is directly proportional to the comb finger overlap length and the square of the applied voltage. In turn, the overlap length is a function of the applied voltage and of the initial geometry, and additional overlap can be created by external shocks or vibrations (figure 4a). Thus, external shocks and vibrations reduce the snapin voltage. A schematic of the modeled combdrive system is shown in figure 4 (b).

Let $l_{\text {overlap }}$ be the initial overlap between the fingers in the absence of any voltage. The initial position of the combdrive is shown in dotted lines. In the presence of a voltage, $V$, across the combdrive, the overlap $l_{V}$ is obtained as

$$
l_{V}=l_{\text {overlap }}+k_{y} V^{2}
$$

where $k_{y}$ is the axial ( $y$-axis) compliance of the combdrive springs with respect to the applied voltage. This holds true until the point of lateral snapping of the combdrive. Let the lateral force required to cause lateral snapping be given by $f_{\text {snap. }}$. Assuming little variation of the overall lateral ( $x$-axis) stiffness of the combdrive spring with axial displacement, the $f_{\text {snap }}$ for any combdrive follows the relationship,

$$
f_{\text {snap }} \propto l_{V-\text { snap -in, comb }} V_{\text {snap-in, comb }}^{2}
$$

where $V_{\text {snap-in,comb }}$ is the snap-in voltage and $l_{V \text {-snap-in,comb }}$ is the corresponding overlap just at the instant just before snap-in. To cause snap-in at a voltage below $V_{\text {snap-in,comb }}$, the additional overlap required can be deduced from (1) and (2) as,

$$
l_{\text {add }}=l_{\text {overlap }}\left[\left(\frac{V_{\text {snap }- \text { in }, \text { comb }}}{V}\right)^{2}-1\right]+k_{y}\left(\frac{V_{\text {snap -in }, \text { comb }}^{4}-V^{4}}{V^{2}}\right)
$$




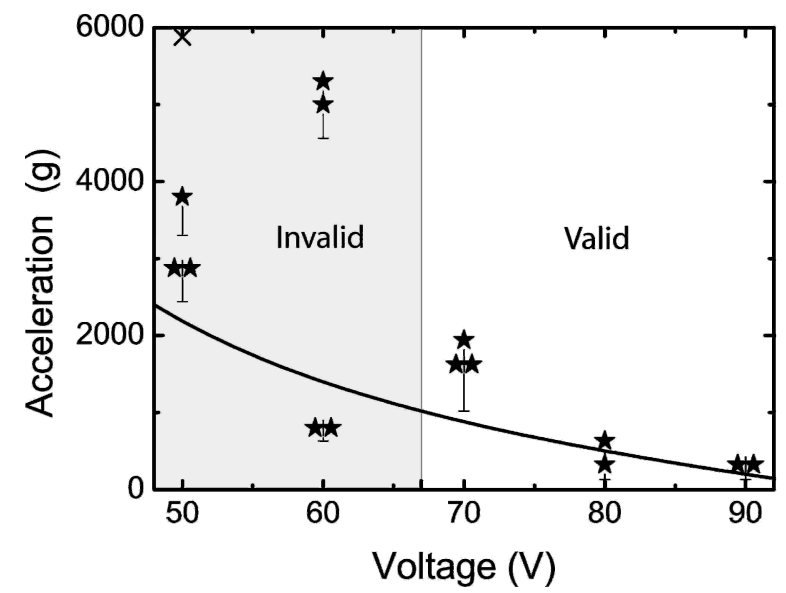

Figure 5. Conditions for axial shock induced lateral snap-in of combdrive at operation voltages below the normal snap-in voltage. The curve shows the predicted conditions. The shaded region indicates the domain where the developed model is not directly applicable since the required overlap exceeds the total length of the combfingers.

In general, (3) can be expressed in terms of the critical acceleration ${ }^{11}$ required to cause failure:

$$
a_{\text {crit }}=\Lambda\left\{l_{\text {overlap }}\left[\left(\frac{V_{\text {snap }-i n, \text { comb }}}{V}\right)^{2}-1\right]+k_{y}\left(\frac{V_{\text {snap }- \text { in }, \text { comb }}^{4}-V^{4}}{V^{2}}\right)\right\}
$$

where $\Lambda$ is a general term representing the expression for converting the displacement to the peak half-sine pulse acceleration, for shock inputs, from the critical shock model and is defined as the peak acceleration of the disturbance required to create a $1 \mathrm{~m}$ displacement and has the units of $\left[\mathrm{s}^{-2}\right]$. The half sine pulse approximation for the acceleration curve closely matches the shock experienced during collisions and will hence be used here ${ }^{11}$.

The input parameters required in (4) were obtained using experimental characterization methods ${ }^{11}$. The mean initial overlap, $l_{\text {overlap }}$, was found to be $3.25 \mu \mathrm{m}$ based on optical profile images. The average lateral combdrive snap-in voltage, $V_{\text {snap-in, }, o m b}$, measured on a few devices is $97.5 \mathrm{~V}$. The axial compliance (with respect to the actuation voltage) $k_{y}$, was obtained as $2 \mathrm{~nm} / \mathrm{V}^{2}$. The value of $\Lambda$ depends on parameters like the shock pulse duration (typically around $200 \mu \mathrm{s}$ ), resonant properties of the in-plane mode (like the quality factor and resonant frequency). The average value of $\Lambda$ is approximately $3.25 \times 10^{7} \mathrm{~g} / \mathrm{m}$ where $\mathrm{g}$ is the acceleration due to gravity ${ }^{11}$. With these parameters, the critical acceleration for the combdrive failures through snap-in can be computed and is shown in figure 5 .

Figure 5 shows that high shock levels lead to reduced snap-in voltages. At zero shock levels, we recover the normal snap-in voltage of $97.5 \mathrm{~V}$. The shaded region shows the portion where the required overlap for failure exceeds the total length of the finger and is hence not valid. The model is then applicable only for voltages above $67 \mathrm{~V}$. For voltages below $67 \mathrm{~V}$, the exact failure levels may vary between devices depending on variables such as the contact surface roughness, geometry, and number of fingers in contact simultaneously ${ }^{11}$. If a collision directly results in welding like defects in sufficiently large number of fingers, the contact may be strong and may lead to permanent defects. In this case the failure acceleration levels are expected to be smaller compared to the predicted values in figure 5. However, if this does not occur, the mobile part may rebound after collision and may be stable; the model in this case does not define the upper limit for failure.

Figure 5 also shows the experimental results of shock testing where the failure levels are indicated by a star ${ }^{11}$. The results in the valid region closely match the prediction curve. The dispersed failure conditions in the shaded region are likely to be due to the inter-device variations. The ' $x$ ' in the dataset indicates a device that did not fail until $\sim 6000 \mathrm{~g}$ half sine pulse shock, which is the upper limit of the shock tester. This is probably an example of the case described previously where collisions simply results in rebound and no failures appear. These results clearly show how devices may not be 
completely robust even when operated at half the actual snap-in voltage in mechanically disturbed conditions. The model, with its close match between predictions and measured failure levels, helps in defining correct operating conditions. Moreover, it can help to avoid performing extensive shock tests to estimate the in-use and transportation failure limits, as a function of the operating voltage.

\section{CONCLUSION}

We have implemented a setup for measuring the dynamics of mobile parts during an ESD event in a MEMS tunable grating. The analysis of the ESD event dynamics brings additional insights into the complex behavior of MEMS devices. A MEMS tunable grating was chosen as test vehicle here. The dynamic analysis allowed one to identify critical parts and help in implementing new protection structures, such as stoppers. Moreover, the analysis revealed subtle mechanisms, not recordable in standard testing, but extremely helpful in preventing new failure paths. The results of ESD dynamics may also act as key inputs for developing phenomenological model, which may greatly benefit the MEMS reliability community.

We also analyzed how combdrive snap-in can occur at operation voltages far below the normal snap-in voltage, if the device is subjected to mechanical shocks or vibrations. We showed how sufficiently large excitations can provide suitable conditions for failures even at operation voltages close to half the actual snap-in voltage. The mechanism for failure has also been proposed here. An analytical model of conditions leading to comb drive snap-in has been developed and the results of the shock tests followed the predicted behavior accurately. The ability to chart out different regimes of the model is essential to understand the conditions for failure and the mechanisms that cause it, and propose possible improvements to the device design. Finally, the model with its good experimental agreement can reduce the need to perform time consuming shock tests to estimate the reliability.

\section{REFERENCES}

[1] Sangameswaran, S., De Coster, J., Linten, D., Scholz, M., Thijs, S., Groeseneken, G. and De Wolf, I., "Invetigating ESD sensitivity in electrostatic SiGe MEMS," J. Micromech. Microeng., 055005 (2010).

[2] Tazzoli, A., Peretti, V., and Meneghesso, G., "Electrostatic discharge and cycling effects on ohmic and capacitive RF-MEMS switches,” IEEE Trans. Dev. Mater. Reliab. 7, 429-37 (2007).

[3] Tanner, D. M., Walraven, J. A., Helgesen, K., Irwin, L. W., Brown, F., Smith, N. F. and Masters N., "MEMS reliability in shock environments," Proc. IEEE Int. Rel. Phys. Symp., 129-138 (2000).

[4] Tanner, D. M., Walraven, J. A., Helgesen, K., Irwin, L. W., Gregory, D. L., Stake, J. R. and Smith, N. F., "MEMS reliability in vibration environment," Proc. IEEE Int. Rel. Phys. Symp., 139-145 (2000).

[5] Van Arsdell, W. W. and Srown, S. B., "Subcritical crack growth in silicon MEMS," J. Microelectromech. Syst. 8, 319-327 (1999).

[6] Modlinski, R., Ratchev, P., Witvrouw, A., Puers, R. and De Wolf, I., "Creep-resistant aluminum alloys for use in MEMS," J. Micromech. Microeng. 15, S165-S170 (2005).

[7] Hartzell, A., da Silva, M. and Shea, H., [MEMS Reliability], Springer (2010).

[8] Tormen, M., Peter, Y. A., Niedermann, P., Hoogerwerf, A., Shea, H. and Stanley, R., "Deformable MEMS grating for wide tunability and high operating speed," Proc. of SPIE 6114, 61140C (2006).

[9] Tormen, M., Peter, Y. A., Niedermann, P., Hoogerwerf, A. and Stanley, R., "Deformable MEMS grating for wide tunability and high operating speed," J. Opt. A: Pure Appl. Opt. 8, S337-S340 (2006).

[10] Sangameswaran, S., De Coster, J., Linten , D., Scholz , M., Thijs, S., Van Hoof, C., De Wolf, I. and Groesenken, G., "Mechanical response of electrostatic actuators under ESD stress," Transducers '09, 21102113 (2009).

[11] Sundaram, S., Tormen, M., Timotijevic, B., Lockhart, R., Overstolz, T., Stanley, R. P. and Shea, H., "Vibration and shock reliability of MEMS: modeling and experimental validation" (under review)

[12] ANSI/ESD STM5.1-2007, [Human body model (HBM): component level], ESD Association (2007). 